\title{
Male Germ Cell-Specific RNA Binding Protein RBMY: A New Oncogene Explaining Male Predominance in Liver Cancer
}

\author{
Daw-Jen Tsuei ${ }^{1}$, Po-Huang Lee ${ }^{2}$, Hsiao-Yu Peng ${ }^{1}$, Shau-Lin Lu', De-Shiuan Su ${ }^{1}$, Yung-Ming Jeng ${ }^{3}$, \\ Hey-Chi Hsu ${ }^{3}$, Shu-Hao Hsu ${ }^{1}$, Jia-Feng $\mathrm{Wu}^{1}$, Yen-Hsuan $\mathrm{Ni}^{1}$, Mei-Hwei Chang ${ }^{1,4 *}$
}

1 Department of Pediatrics, College of Medicine, National Taiwan University, National Taiwan University Hospital, Taipei, Taiwan, 2 Department of Surgery, College of Medicine, National Taiwan University, National Taiwan University Hospital, Taipei, Taiwan, 3 Department of Pathology, College of Medicine, National Taiwan University, National Taiwan University Hospital, Taipei, Taiwan, $\mathbf{4}$ Hepatitis Research Center, College of Medicine, National Taiwan University, National Taiwan University Hospital, Taipei, Taiwan

\begin{abstract}
Male gender is a risk factor for the development of hepatocellular carcinoma (HCC) but the mechanisms are not fully understood. The RNA binding motif gene on the Y chromosome (RBMY), encoding a male germ cell-specific RNA splicing regulator during spermatogenesis, is aberrantly activated in human male liver cancers. This study investigated the in vitro oncogenic effect and the possible mechanism of RBMY in human hepatoma cell line HepG2 and its in vivo effect with regards to the livers of human and transgenic mice. RBMY expression in HepG2 cells was knocked down by RNA interference and the cancer cell phenotype was characterized by soft-agar colony formation and sensitivity to hydrogenperoxide-induced apoptosis. The results revealed that RBMY knockdown reduced the transformation and anti-apoptotic efficiency of HepG2 cells. The expression of RBMY, androgen receptor (AR) and its inhibitory variant AR45, AR-targeted genes insulin-like growth factor 1 (IGF-1) and insulin-like growth factor binding protein 3 (IGFBP-3) was analyzed by quantitative RT-PCR. Up-regulation of AR45 variant and reduction of IGF-1 and IGFBP-3 expression was only detected in RBMY knockdown cells. Moreover, RBMY positive human male HCC expressed lower level of AR45 as compared to RBMY negative HCC tissues. The oncogenic properties of RBMY were further assessed in a transgenic mouse model. Liver-specific RBMY transgenic mice developed hepatic pre-cancerous lesions, adenoma, and HCC. RBMY also accelerated chemical carcinogen-induced hepatocarcinogenesis in transgenic mice. Collectively, these findings suggest that $Y$ chromosomespecific RBMY is likely involved in the regulation of androgen receptor activity and contributes to male predominance of HCC.
\end{abstract}

Citation: Tsuei D-J, Lee P-H, Peng H-Y, Lu S-L, Su D-S, et al. (2011) Male Germ Cell-Specific RNA Binding Protein RBMY: A New Oncogene Explaining Male Predominance in Liver Cancer. PLoS ONE 6(11): e26948. doi:10.1371/journal.pone.0026948

Editor: John D. Minna, University of Texas Southwestern Medical Center at Dallas, United States of America

Received June 27, 2011; Accepted October 6, 2011; Published November 4, 2011

Copyright: (c) 2011 Tsuei et al. This is an open-access article distributed under the terms of the Creative Commons Attribution License, which permits unrestricted use, distribution, and reproduction in any medium, provided the original author and source are credited.

Funding: This work was supported by grants NHRI-EX93-117BN, NHRI-EX95 $(96,97,98)-9418 \mathrm{BI}$ from the National Health Research Institute of Taiwan. The funders had no role in study design, data collection and analysis, decision to publish, or preparation of the manuscript.

Competing Interests: The authors have declared that no competing interests exist.

* E-mail: changmh@ntu.edu.tw

\section{Introduction}

Hepatocellular carcinoma (HCC) is one of the leading cancers in the world [1]. The major identified risk factors include hepatitis $\mathrm{B}$ virus (HBV) or hepatitis $\mathrm{C}$ virus (HCV) infection, exposure to aflatoxins, and male gender [2]. The male-to-female ratio of HCC reportedly averages 4-5:1 and is particularly higher in HBVrelated HCG (5-11:1) [3]. Epidemiological studies showed that an elevated level of serum testosterone was significantly associated with an increased risk of HCG in male HBV carriers [4]. However, male predominance with a ratio of 3-4:1 is also observed in childhood HCC with early onset, as young as $<10$ years of age before puberty, and cannot be explained directly by androgen effect $[5,6]$.

The androgen receptor (AR) has been shown to contribute to the male preference of hepatocarcinogenesis in $\mathrm{HBV}$ and $\mathrm{HCV}$ carriers $[7,8]$. The AR protein mediates the action of androgens and can promote the development of male HCC in mice [9]. After binding to ligands, AR forms homodimers and activates the transcription of target genes such as insulin-like growth factor 1 (IGF-1) and insulin-like growth factor binding protein 3 (IGFBP-3) [10,11]. The AR function is regulated by co-activators or corepressors [12], the inhibitory isoform AR45 [13], and shorter CAG repeats (that leads to higher AR activity) in the first exon of the AR gene [14]. The HBV X protein is a well-known AR coactivator $[15,16]$ and was reported to contribute to male predominance in HBV-related human male HCG [17]. In nonHBV HCC, other unknown gender-specific factors promoting HCC formation in males have become a major concern.

Dysregulation of Y chromosome-specific genes has been found in male HCG, but their roles in the male predominance of HCC so far have not been addressed [18,19]. The RNA-binding motif gene on the $\mathrm{Y}$ chromosome (RBMY) is a male germ cell-specific expression gene containing RNA recognition motifs at the $\mathrm{N}$ terminus [20]. Its expression is confined to the nuclei of male germ cells and its deletion may cause the arrest of germ cells at the 
meiotic stage of spermatogenesis [21]. RBMY functions as a male germ cell-specific splicing regulator by modulating the activity of constitutively expressed splicing factors [22,23]. Its aberrant activation is detected in about $1 / 3$ of male HCG and hepatoblastoma tumor tissues, but not in paired non-tumor liver tissues, female HCC, or other types of cancers [24]. Though RBMY can interact with AR co-activator Sam68 [25,26], its role in the AR signaling pathway has not yet been reported.

The present study aimed to evaluate the transformation and antiapoptotic efficacy of RBMY in human hepatoma cells, and the hepatocarcinogenic efficacy of RBMY in transgenic mice, and to explore its possible underlying molecular mechanisms. Our results in-vitro, in-vivo, and in clinical human male HCC tissues suggest that RBMY may enhance AR activity and hepatocarcinogenesis by reducing the expression of $\mathrm{AR}$ inhibitory variant $\mathrm{AR} 45$.

\section{Materials and Methods}

\section{Human tissue samples}

Paired tumor and non-tumor liver tissues were collected from 66 surgical HCG male patients (44 HBV-related, $10 \mathrm{HCV}$-related, 5 $\mathrm{HBV} / \mathrm{HCV}$-related, and 7 non-HBV/HCV-related) at the National Taiwan University Hospital (NTUH) in 2007. All clinical samples were obtained following approval of the Research Ethics Committee of the NTUH (NTUHREC approval No. 9361701139).

\section{Cell culture and transfection}

The human hepatocellular carcinoma cell lines HepG2 and Hep3B were originally obtained from the Bioresource Collection and Research Center (BCRG, Taiwan). HepG2, Hep3B and Huh7 (from Dr. Hui-Lin Wu of NTUH Hepatitis Research Center) cells were grown in Dulbecco's modified Eagle's medium (DMEM) supplemented with 10\% fetal calf serum (HyClone, Logan, UT). Transfections were performed using Lipofectamine (Invitrogen/Life Technologies, Carlsbad, CA) according to the manufacturer's protocol.

\section{RNA interference}

The pSUPER-based strategy was used to knockdown RBMY expression. RBMY small hairpin RNA (shRNA) was generated by ligating three 19-nucleotide sequences specific for RBMY SRGY region (exons 7-10) into the vector (OligoEngine, Inc., Seattle, WA). The sequences were shown in Table S1. Cells were grown to $80 \%$ confluence for transfection and transfected cells were selected with $300 \mu \mathrm{g} / \mathrm{ml}$ geneticin.

\section{Semi-quantitative RT-PCR and quantitative PCR}

Total RNA was extracted using RNeasy Mini kit (QIAGEN, $\mathrm{GmbH}$, Hilden, Germany) and subjected to reverse transcription using SuperScript III Reverse Transcriptase (Invitrogen). The primer sequences and reaction conditions were shown in Table S1. Quantitative PCR was performed using the TaqMan Gene Expression Assay mix for target RBMY (Hs00359074_ml) or endogenous control hypoxanthine phosphoribosyl transferase 1 (Hs99999909_ml) (Applied Biosystems, Foster City, CA). Quantitative PCR for AR, AR45, IGF-1, IGFBP-3, and endogenous control S26 was performed using the MasterMix Plus for SYBR Green (Applied Biosystems). Amplification signals were detected by an ABI prism7700 or 7500 Fast Real-Time PCR System (Applied Biosystems).

\section{Soft agar and survival assays}

For soft agar assay, 2500 cells per well in growth medium containing $0.35 \%$ agarose were placed on 6 -well plates with $0.5 \%$ agarose base.
After 14 days of incubation, the colonies were stained with $0.005 \%$ crystal violet at room temperature for $1 \mathrm{hr}$ and counted for each plate.

For survival assay, the HepG2 cells transfected with shRNA plasmids were treated with 0.5 or $0.75 \mathrm{mM}$ hydrogen peroxide for $24 \mathrm{hr}$ to induce apoptosis. Cell viability was measured using the Cell Proliferation Kit I (MTT) (Roche Diagnostics, Germany). Absorbance was measured at 590/690 nm using a MRX microplate reader (Dynex Technologies, Inc., VA) and cell survival was expressed as absorbance relative to that of untreated control.

\section{Determination of androgen receptor CAG-repeat length in human HCC tissues}

The genomic region containing the CAG trinucleotide repeat was PCR-amplified and labelled with FAM, subjected to the ABI 3700 Genetic Analyzer, and scored using the GeneMapper software (Applied Biosystems). The standard curve to calculate the CAG repeat number was based on four control samples with CAG repeat numbers ranging from 17 to 35 . Sequencing analysis was performed on HCC tissues from 66 study subjects with CAG repeat numbers out of the standard curve.

\section{Establishment of RBMY transgenic mice and follow-up histopathology}

The RBMY coding sequence was amplified and sub-cloned into the pBS-HCRHPI-A vector with a liver-specific $\alpha 1$-antitrypsin promoter. The construct was digested with SpeI to generate a transgene fragment for injection into the pro-nuclei of fertilized eggs of FVB/N mice. The Institutional Animal Care and Use Committee of the College of Medicine and College of Public Health, National Taiwan University approved the animal care and experimental procedures (IACUG approval No. 20060217).

The mice were sacrificed at 4-24 months old. Their livers were fixed in $10 \%$ neutral-buffered formalin or embedded in OCT compound (Sakura Finetek, Torrance, CA). Paraffin sections were stained with hematoxylin and eosin for histopathology examination, while frozen sections were stained with oil red $\mathrm{O}$ to detect fatty droplet accumulation. The severity of steatosis was estimated by the percentage of positive staining using a morphologic semiquantitative method and classified into grade $1(<33 \%)$, grade 2 (33-66\%), or grade $3(>66 \%)$ as described previously [27].

\section{Diethylnitrosamine treatment and histopathology in mice}

In the chemical carcinogen model, 14-day old RBMY transgenic or control mice randomly received a single intraperitoneal injection of diethylnitrosamine (DEN) (Sigma-Aldrich, Inc., St Louis, MO) at $10 \mathrm{mg} / \mathrm{kg}$ of body weight or saline solution of equal volume, respectively. The mice were sacrificed at 26 and 34 weeks post-treatment. In addition, earlier sacrifice at 14 weeks was specifically adopted for male mice that were reportedly highly susceptible to DEN-induced hepatocarcinogenesis. Measurements of tumor masses visible on the liver surface were recorded. Microscopic lesions were counted from two representative sections (50- $\mu \mathrm{m}$ distance) of each mouse liver.

\section{Immuno-histochemistry}

Immuno-histochemical staining for the RBMY antigen was performed on frozen liver sections. After antigen retrieval and quenching of endogenous peroxidation, the rabbit anti-SRGY antibody [24] was incubated with sections at 1:400 dilution overnight at $4^{\circ} \mathrm{C}$. HRP-DAB was used as a detection system (R\&D Systems, Inc., Minneapolis, MN) and counter-stained by hematoxylin. 


\section{Western blotting}

The extraction of total protein, Western blot analysis, and antiSRGY antibody preparation were performed as described previously [24]. Briefly, $50 \mu \mathrm{g}$ of total protein extracted from individual mouse liver tissue were separated by electrophoresis using standard SDS-PAGE. The blots were incubated with rabbit polyclonal anti-SRGY antibodies at 1:5000 dilution and mouse monoclonal anti-glyceraldehyde-3-phosphate dehydrogenase (GAPDH) antibodies (Abcam, Cambridge, UK) at 1:1000 dilution.

\section{Statistical analysis}

Statistical differences in the association between AR-CAG repeats and RBMY, viability assay, fold change in RNA expression, and DEN treatment analysis were analyzed by twotailed Student's $t$-test. Difference of steatosis incidences between the transgenic and control mice was analyzed by Chi-square test. Soft-agar colony assay was evaluated by one-way ANOVA followed by $t$-test. After normalization against S26, relative values of AR and AR45 expression were analyzed by Student's $t$-test with unequal variance. A $p<0.05$ was considered statistically signifi- cant, while a $p$ value between 0.05 and 0.1 was considered as having a trend of difference.

\section{Results}

RBMY knockdown reduced the transformation and anti-apoptotic efficiencies of HepG2 cells

Natively expressed RBMY in human hepatoma cell line HepG2 was interfered by shRNA specifically targeting to the SRGY domain of RBMY. Quantitative RT-PCR showed that $>95 \%$ of RBMY transcripts were inhibited in pSUPER-680 and pSUPER914 transfected HepG2 cells, whereas pSUPER-778 had no knockdown effect compared to vector transfected cells (Fig. 1A). Immuno-histochemistry analysis further confirmed efficient knockdown of RBMY in pSUPER-680 and pSUPER-914 transfected cells (Fig. 1B). The vector-only transfectants expressed similar levels of RBMY compared to parental HepG2 cells and were therefore used as controls for in vitro cell experiments.

The anchorage independence assay showed that RBMYexpressing HepG2 cells had stronger clonogenic ability compared
A

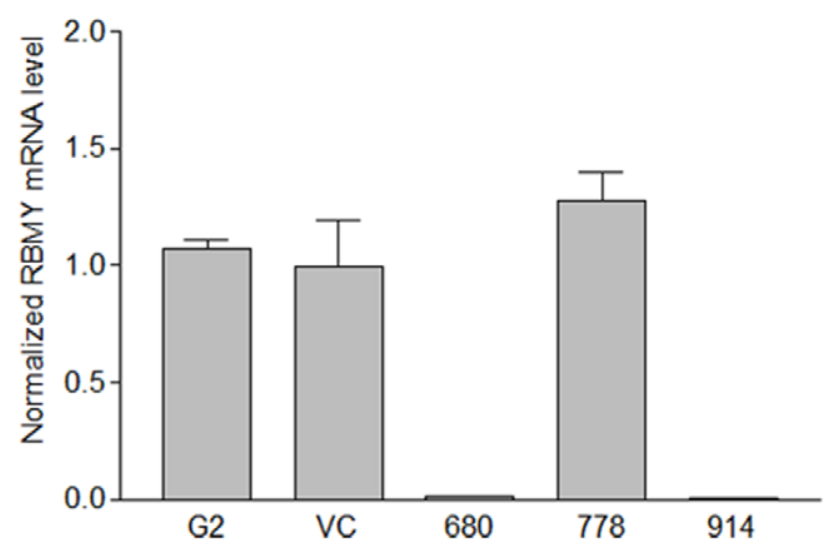

$\mathrm{B}$

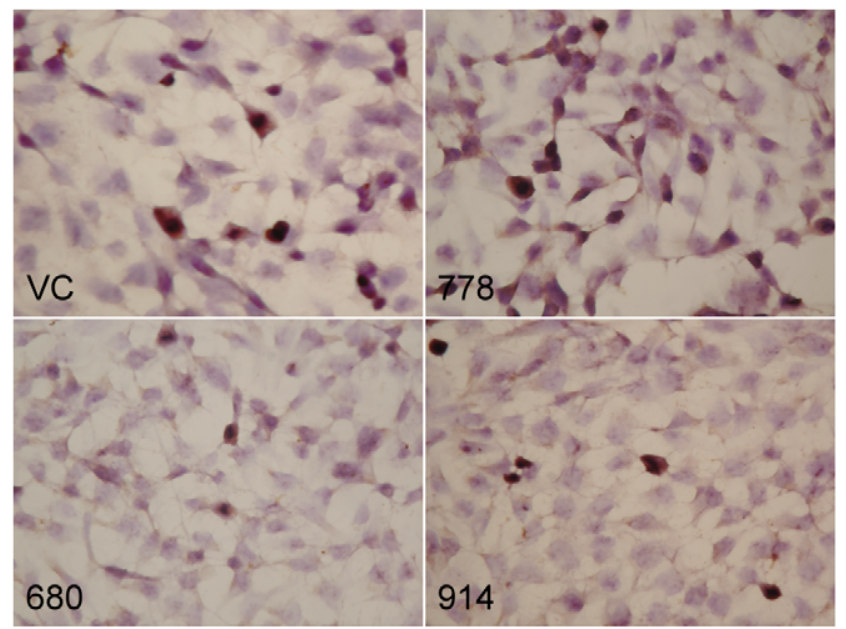

C
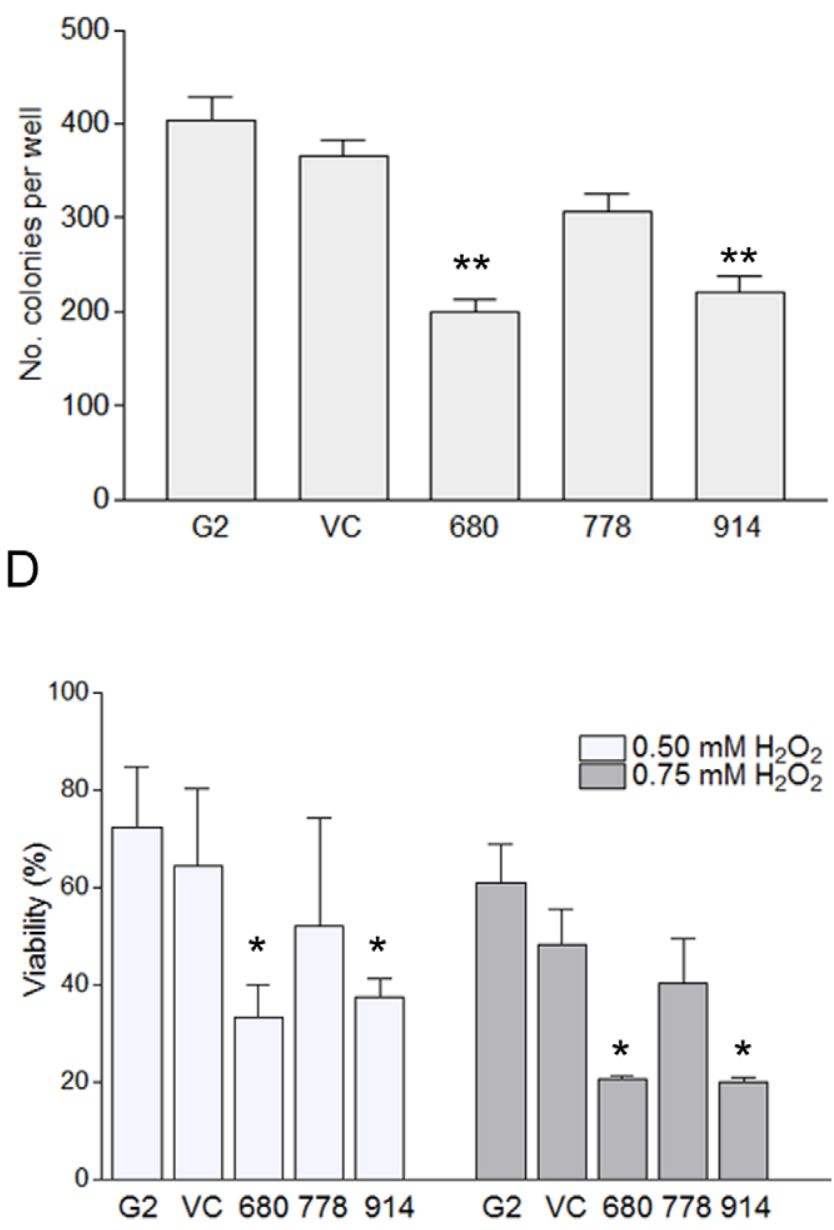

Figure 1. RBMY knockdown reduced the transformation and anti-apoptotic efficiencies of HepG2 cells. (A) Quantitative RT-PCR analysis of RBMY in parental (G2), vector plasmid (VC), pSUPER-680, pSUPER-778, and pSUPER-914 plasmids transfected HepG2 cells. (B) Immunohistochemical staining of HepG2 and the corresponding transfectants for RBMY protein. (C) Colony formation of parental or transfected HepG2 cells on soft agar. (D) Percentage of cell viability by $\mathrm{MTT}$ assay post 0.5 or $0.75 \mathrm{mM}$ hydrogen peroxide $\left(\mathrm{H}_{2} \mathrm{O}_{2}\right)$ treatment. Data were presented as mean \pm SD in four independent experiments. ${ }^{*} p<0.05 ;{ }^{* *} p<0.01$.

doi:10.1371/journal.pone.0026948.g001 
to RBMY knockdown cells $(p<0.0001$, one-way ANOVA). The colony numbers of pSUPER-680 and pSUPER-914 transfected cells on soft agar were reduced by $45 \%$ and $40 \%$, respectively, compared to empty vector transfectants (Fig. 1G). Colony formation of RBMY-expressing pSUPER-778 transfectants was also significantly higher than knockdown transfectants (680 vs. $778, p<0.0001 ; 914$ vs. $778, p=0.001$ ).

The anti-apoptotic abilities of RBMY-expressing and knockdown HepG2 transfectants were analyzed by survival assay after hydrogen peroxide treatment. Compared to empty vector transfectants, the viability of pSUPER-680 shRNA transfectants with 0.5 or $0.75 \mathrm{mM}$ hydrogen peroxide treatment was reduced by $46 \%$ and $53 \%(p<0.05)$, respectively, and those of pSUPER914 transfectants were reduced by $34 \%$ and $54 \%(p<0.05)$, respectively (Fig. 1D). The viability of RBMY-expressing transfectants pSUPER-778 was $16-19 \%$ lower than that of vector transfectants $(p=0.5)$.

RBMY knockdown increased the expression of inhibitory androgen receptor variant AR45 and decreased AR trans-activation activity, while RBMY overexpression suppressed AR45 levels

It has been reported that Sam68, as an alternative splicing regulator and interacting protein of RBMY, might modulate ARregulated transcriptional activity in prostate cancer cells [25]. To examine whether the expression ratio of $\mathrm{AR}$ and its inhibitory isoform AR45 were affected by RBMY, semi-quantitative RTPCR was performed for RBMY-expressing or knockdown HepG2 cells. Densitometry analysis showed a three-fold increase of AR45 levels in RBMY knockdown transfectants (pSUPER-680 and pSUPER-914) compared to vector-only (VG) transfectants $(p<0.05)$ (Fig. 2A). AR45 levels in parental HepG2 cells and pSUPER-778 transfectants were similar to those in vector transfectants. There was no significant change in AR levels between RBMY-expressing and knockdown cells. The AR/AR45 ratios in RBMY knockdown transfectants (pSUPER-680 and pSUPER-914) were reduced by 2- to 2.5-fold compared to vectoronly (VC) transfectants (Fig. 2A).

As AR45 was reported to suppress AR trans-activation activity [13], the expression of AR target genes IGF-1 and IGFBP-3 was assessed and compared between RBMY-expressing and knockdown HepG2 cells. Quantitative RT-PCR analysis revealed that RBMY knockdown cells expressed high levels of AR45 but significantly reduced IGF-1 $(p<0.05)$ and IGFBP-3 $(p<0.01)$ levels (Fig. 2B), showing that RBMY knockdown correlated with enhanced AR45 expression and repressed AR activity.

Furthermore, the suppression of AR45 expression by overexpressing RBMY was also demonstrated in Hep3B and Huh7 cells. AR45 levels were reduced to $42 \%$ in RBMY transfected Hep3B and $60 \%$ in Huh7 cells $(p<0.05)$ (Fig. 2C). There was no significant change in AR levels between RBMY-transfected and vector-transfected cells.

\section{RBMY expression correlated with reduced AR45 levels} and shorter AR-CAG repeats in human male HCC tissues

Based on the above mentioned results showing that RBMY expression was associated with lower AR45 levels in HepG2 cells, $\mathrm{AR}$ and AR45 expressions were further compared in RBMYexpressing and non-expressing human male HCC tissues. Semiquantitative RT-PCR showed that RBMY was detected in $35 \%$ $(23 / 66)$ male HCC tumor tissues, which were confirmed by the expression of tumor marker alpha fetoprotein (Fig. 2D). Immunohistochemical staining confirmed the nuclear expression of RBMY only in HCC tumor tissues (Fig. 2E). The positive rate of RBMY transcripts was 36\% (16/44) in HBV-related HCC, 40\% (4/10) in HCV-related HCC, 20\% (1/5) in HBV/HCV-related HCG, and $29 \%(2 / 7)$ non-viral related HCG. There were no detectable RBMY transcripts or proteins in the non-tumor portions of the 66 HCC liver tissues.

The expression of $\mathrm{AR}$ and $\mathrm{AR} 45$ variant in seven RBMYexpressing and five non-expressing male human HCG tissues was determined by $2 \exp ^{(-\Delta \Delta \mathrm{Ct})}$ method for relative quantification. The mRNA levels in HCC tissues were normalized against the internal control S26 and compared to the levels in vector-only HepG2 transfectants. Significantly lower levels of AR45 were detected in RBMY-expressing male HCC compared to non-RBMY expressing HCG tissues $(p<0.05)$ (Fig. 2F), reflecting RBMY suppression on AR45 transcription. There was no significant difference of AR levels between RBMY-expressing and non-expressing HCC tissues.

To further assess the association between RBMY and AR activity, the CAG-repeat length of the AR gene, an AR activityassociated factor, was determined in RBMY-expressing or nonexpressing male human HCC tissues. The mean CAG-repeat length in $49 \mathrm{HBV}$-related male HCG tissues (RBMY positive/ negative $=17 / 32$ ) was significantly shorter in those with than in those without RBMY expression (21.0 22.8 vs. $22.9 \pm 2.8, p<0.05)$. The association of CAG-repeat length and RBMY expression revealed a trend of difference $(21.4 \pm 2.54$ vs. $22.7 \pm 2.75, p=0.064)$ when non-HBV HCG tissues were included (RBMY positive/ negative $=23 / 43)$.

\section{RBMY transgenic mice developed hepatic fatty change and neoplastic lesions}

Transgenic mice with liver-specific expression of RBMY were established (Fig. 3A). Western blot showed low RBMY expression in transgenic founders RF1 and RF2, but high RBMY level in RF4 and RF6 (Fig. 3B). There was no transgenic RBMY detected in the brain, heart, kidney, lung, spleen, stomach, or testis by RTPCR (Fig. 3C), indicating a liver-specific expression of RBMY in transgenic mice. Quantitative RT-PCR results further supported RF4 as a high expression founder (Fig. 3D). IHC staining showed that RBMY was mainly located in the nucleus of transgenic mice liver tissues (Fig. 3E).

Hepatocellular changes were analyzed from liver sections of 79 transgenic mice and 30 control FVB/N mice. High RBMYexpressing RF4 founders (RF4-19 and RF4-21) displayed more severe fatty deposit than low RBMY-expressing RF2 founders (RF2-62 and RF2-390) (Fig. 3F). In RBMY transgenic mice, the incidence of developing moderate-to-severe hepatic steatosis was $60-89 \%$ (average $73 \%$ ), which was $\sim 2.5$-fold higher than that of the control group (average 30\%, $p<0.001$ ) (Table 1). RBMY expression did not increase fibrosis or cirrhosis in the transgenic mice model.

Among the 45 transgenic mice which were older than 15 months, three of them developed hepatic cancerous lesions (Table 1). A nodule (1 $\mathrm{mm}$ in diameter) was found in the right lobe of RF1-278 mouse (15 months) and histological examination revealed a pre-neoplastic lesion (Fig. 4A-C). The RF4-2 1 mouse (24 months) had an adenoma nodule (4 $\mathrm{mm}$ in diameter) in the right lobe (Fig. 4D-F) while RF2-390 (21 months) had moderately differentiated $\mathrm{HCG}(6 \mathrm{~mm}$ in diameter) in the median lobe (Fig. 4G-I). Medium-to-severe fatty changes were observed in the tumor parts of the three mice. None of 17 control mice older than 15 months developed hepatic cancerous lesions. 
A

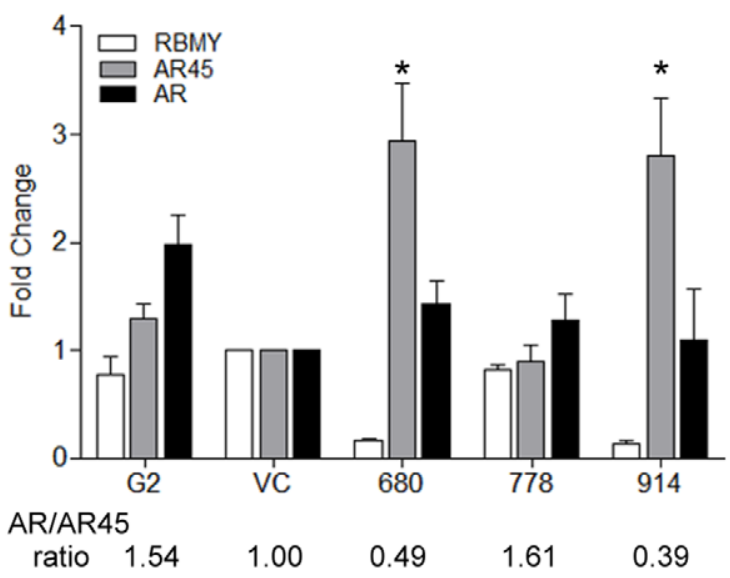

B

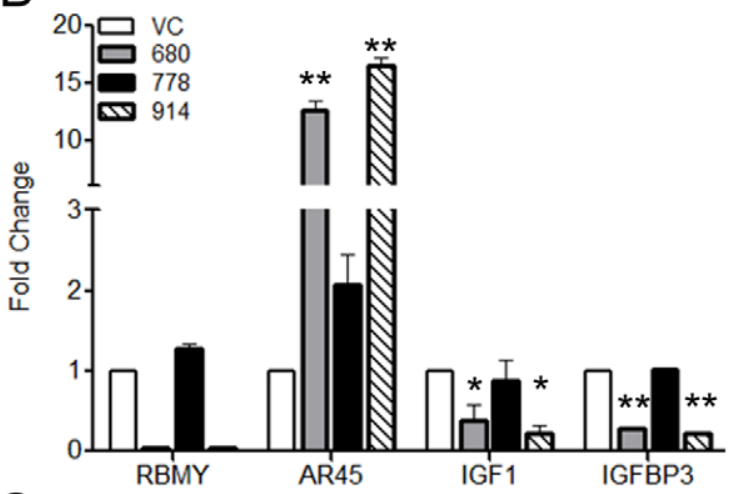

C

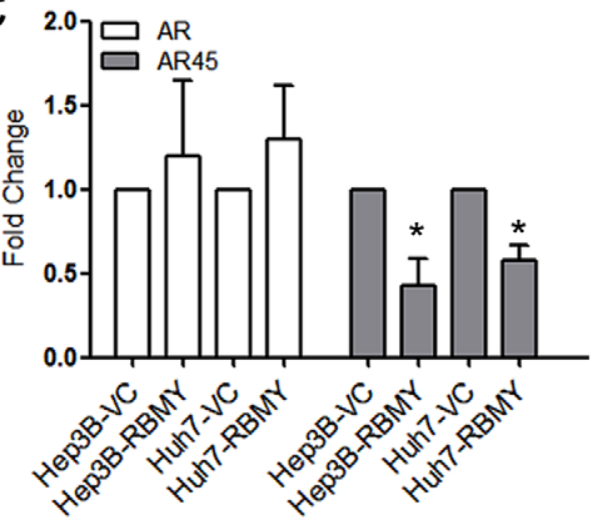

D

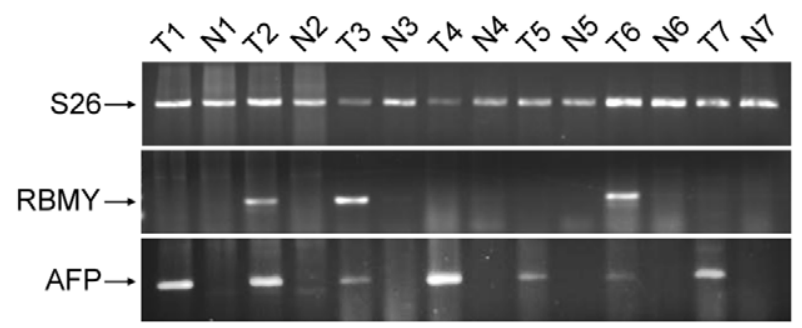

E

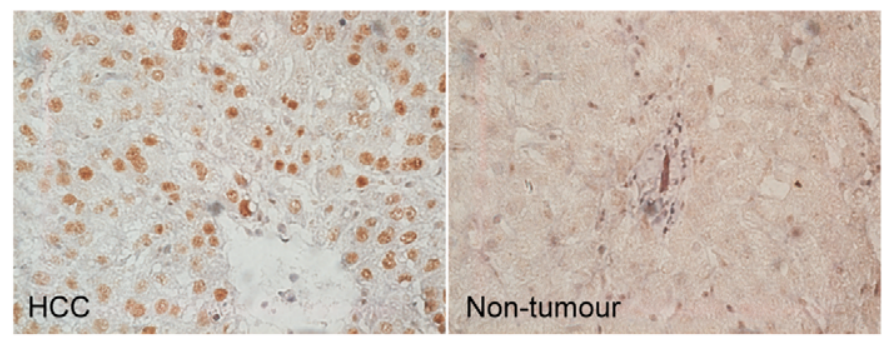

$F$

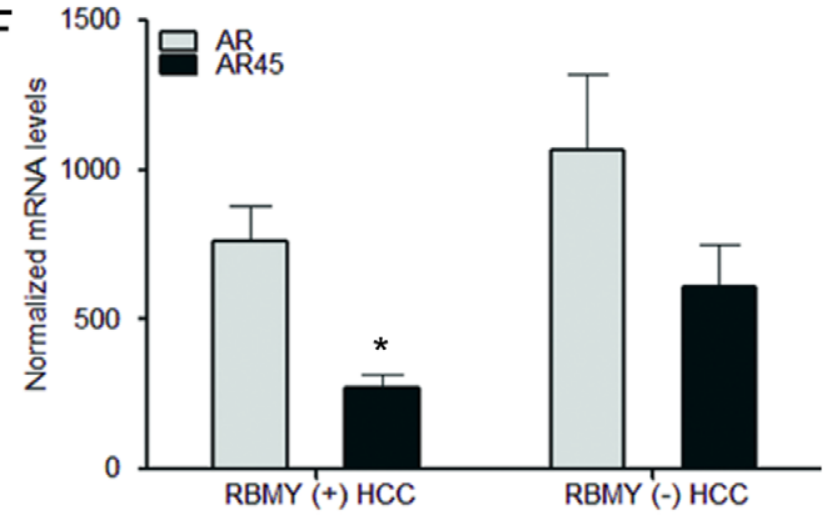

Figure 2. RBMY expression correlated with reduced androgen receptor AR45 variant in human hepatoma cell lines and male HCC tissues. (A) Semi-quantitative RT-PCR and densitometric analysis of RBMY, AR and AR45 in parental (G2), vector plasmid (VC), pSUPER-680, pSUPER778, and pSUPER-914 plasmids transfected HepG2 cells. (B) Quantitative RT-PCR analysis of RBMY, AR45, IGF-1 and IGFBP-3 in RBMY knockdown transfectants (680 and 914) and non-knockdown transfectants (VC and 778). Values were normalized against the internal control S26. Data are mean \pm SD folds over vector control (VC). (C) Quantitative RT-PCR analysis of AR and AR45 in vector control or RBMY transfected Hep3B and Huh7 cells. Data are mean \pm SD folds over vector control (VC). (D) Semi-quantitative RT-PCR analysis of RBMY in the tumor (T) and non-tumor (N) parts of human male HCCs. S26 is an internal control and alpha fetoprotein (AFP) as a tumor marker. (E) Immuno-histochemical staining of nuclear RBMY protein in HCC tissues only $(\times 400)$. (F) AR and AR45 mRNA expression in 7 RBMY-expressing and 5 non-expressing human male HCC tissues. The results were the mean of three different experiments. ${ }^{*} p<0.05 ;{ }^{*} p<0.01$.

doi:10.1371/journal.pone.0026948.g002

\section{RBMY accelerated diethyl nitrosamine-induced hepatocarcinogenesis}

The tumor-promoting effects of RBMY were further assessed in the chemical carcinogenesis model. Because of the high susceptibility of male mice to DEN, male groups were sacrificed at 14 weeks post-treatment. A higher incidence of cancerous lesions was observed in transgenic male mice as compared to that in the control group $(12 / 14$ vs. $5 / 12, p<0.05)$. At 34 weeks posttreatment, male RBMY transgenic mice developed more tumors with diameter $>3 \mathrm{~mm}$ compared to the control group (52 vs. 19, $p<0.05$ ) (Table 2). There was also a higher incidence of trabecular cancerous lesions in female transgenic mice compared to the 


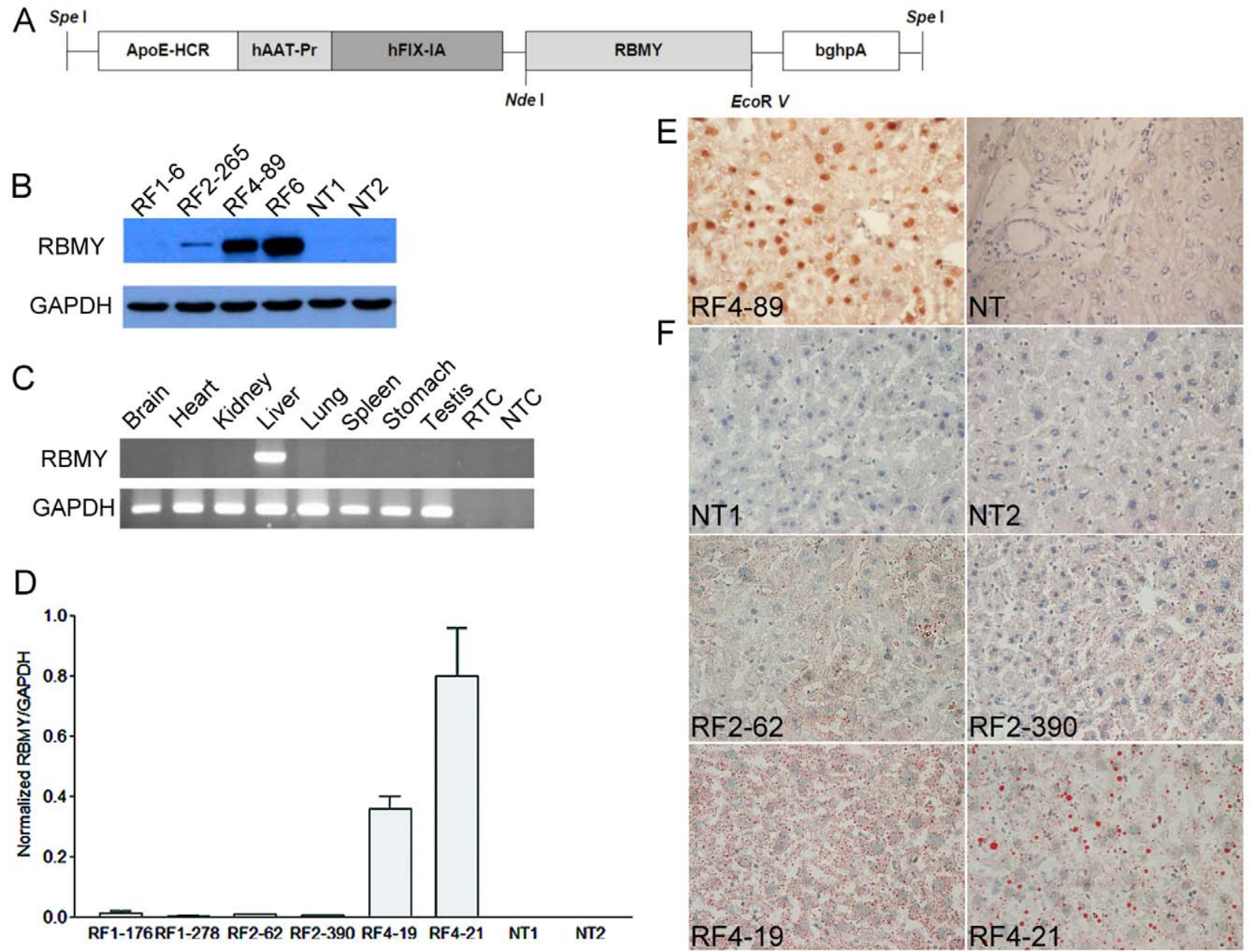

Figure 3. Characterization of RBMY transgenic mice. (A) Genetic map of the $4.2 \mathrm{~kb}$ RBMY transgene, including a hepatic locus control region from the apolipoprotein E gene (ApoE-HCR), liver-specific $\alpha 1$-antitrypsin promoter (hAAt-Pr), truncated factor IX intron (hFIX-IA), and bovine growth hormone polyadenylation signal (bghpA). (B) Western blot analysis of RBMY prepared from the livers of individual transgenic (RF1-6, RF2-265, RF4-89, RF6) and control (NT1, NT2) mice. (C) Liver-specific expression of RBMY in transgenic mice by RT-PCR using GAPDH as an internal control. Reactions without template RNA (RTC) or CDNA (NTC) were negative controls. (D) Expression of RBMY in transgenic (RF1-176, RF1-278, RF2-62, RF2-390, RF4-19, RF4-21) and control (NT1, NT2) mice by quantitative RT-PCR. (E) Immuno-histochemical staining of transgenic (RF4-89) and control (NT) mice liver tissues for RBMY. Transgenic mouse showed nuclear staining for RBMY $(\times 400)$. (F) Oil red staining showed hepatic fatty changes in control mice (NT1 and NT2), low RBMY (RF2-62 and RF2-390) and high RBMY (RF4-19 and RF4-21) transgenic mice $(\times 400)$. doi:10.1371/journal.pone.0026948.g003

control group at 26 weeks $(7 / 9$ vs. $1 / 10, p<0.01)$ and 34 weeks $(9 /$ 10 vs. $3 / 13, p<0.01$ ) post DEN treatment (Table 2$)$.

\section{Discussion}

RBMY is regarded as a testis-specific splicing factor in spermatogenesis $[22,23]$. It has been reported to be expressed in more than one-third of human male HCG tissues [24]. This study further demonstrates that RBMY knockdown correlates with increased AR45 variant expression and reduced anchorage independent growth and anti-apoptotic abilities of human hepatoma cell line HepG2. AR45 isoform is reported to act as a dominant-negative inhibitor of $\mathrm{AR}$ function through the formation of AR-AR45 heterodimers [13]. We also demonstrate that RBMY knockdown reduces AR trans-activation activity in HepG2 cells. Therefore, RBMY may function as a male-specific oncogene and increase the risk of human male hepatocarcinogenesis through regulation of $\mathrm{AR}$ gene expression and activity.
The AR gene, rather than androgen, has been shown to play a key role in the male predominance of $\mathrm{HCC}$ in a transgenic $\mathrm{HBV}$ mouse model [17]. Human AR is composed of $\mathrm{N}$ terminal transactivation domain, a central DNA-binding domain, and a $\mathrm{C}$ terminal ligand-binding domain. AR45, a naturally occurring variant form of human androgen receptor, lacks the N-terminal domain required for full ligand activated transcriptional activity [13]. The inverse association of AR and AR45 expression has been observed in human heart and muscle tissues with the highest levels of AR45 and the lowest levels of AR [13]. In this study, RBMY knockdown increases expression of AR45 in human hepatoma HepG2 cell line, while RBMY overexpression reduces AR45 levels in Hep3B and Huh7 cell lines. Moreover, RBMY-positive human male HCC tissues express lower AR45 levels compared to RBMYnegative HCG. The down-regulation of AR target genes IGF-1 and IGFBP-3 in RBMY knockdown HepG2 cells further illustrates the enhancing effect of RBMY on AR trans-activation activity. HBV X protein has been reported to function as a virus-encoding AR co-activator that significantly contributes to the male 
Table 1. Summary of histological findings of RBMY transgenic and control mice.

\begin{tabular}{|c|c|c|c|c|c|}
\hline \multirow{3}{*}{ Mice } & \multirow{3}{*}{$\frac{\text { Gender }}{\text { (Mice No.) }}$} & \multirow{3}{*}{$\frac{\text { Age }}{\text { (days) }}$} & \multirow{3}{*}{$\begin{array}{l}\text { Liver/body } \\
\text { weight } \\
(\times 100)\end{array}$} & \multirow{3}{*}{$\begin{array}{l}\text { Incidence of } \\
\text { grade } 2 / 3 \\
\text { steatosis }\end{array}$} & \multirow{3}{*}{$\begin{array}{l}\text { Incidence of } \\
\text { pre-neoplastic lesions } \\
\text { ladenoma/HCC }\end{array}$} \\
\hline & & & & & \\
\hline & & & & & \\
\hline \multirow[t]{2}{*}{ TgRBMY } & $F(37)$ & $496.4 \pm 158.3$ & $4.49 \pm 0.97$ & $89 \%{ }^{* *}$ & $1 / 37$ \\
\hline & M (42) & $474.2 \pm 154.0$ & $4.69 \pm 1.02$ & $60 \%{ }^{*}$ & $2 / 42$ \\
\hline \multirow[t]{2}{*}{ NT } & $F(15)$ & $439.9 \pm 191.8$ & $5.09 \pm 1.05$ & $40 \%$ & $0 / 15$ \\
\hline & M (15) & $433.5 \pm 185.1$ & $5.21 \pm 1.14$ & $20 \%$ & $0 / 15$ \\
\hline TgRBMY & $\mathrm{F}+\mathrm{M}(79)$ & $484.6 \pm 155.4$ & $4.60 \pm 1.00$ & $73 \%{ }^{* *}$ & $3 / 79$ \\
\hline NT & $\mathrm{F}+\mathrm{M}(30)$ & $436.7 \pm 185.3$ & $5.15 \pm 1.08$ & $30 \%$ & $0 / 30$ \\
\hline
\end{tabular}

The liver frozen sections from control (NT) and RBMY transgenic (TgRBMY) mice were stained with oil red $\mathrm{O}$ and classified to grade 2 and 3 with $33 \sim 66 \%$ and $>66 \%$ positive stain, respectively.

The paraffin sections were stained with hematoxylin and eosin for histopathology examination

Values are shown as mean $\pm \mathrm{SD}$.

${ }^{*} p<0.05$;

** $p<0.001$; transgenic vs. control mice; by Chi-square test.

doi:10.1371/journal.pone.0026948.t001

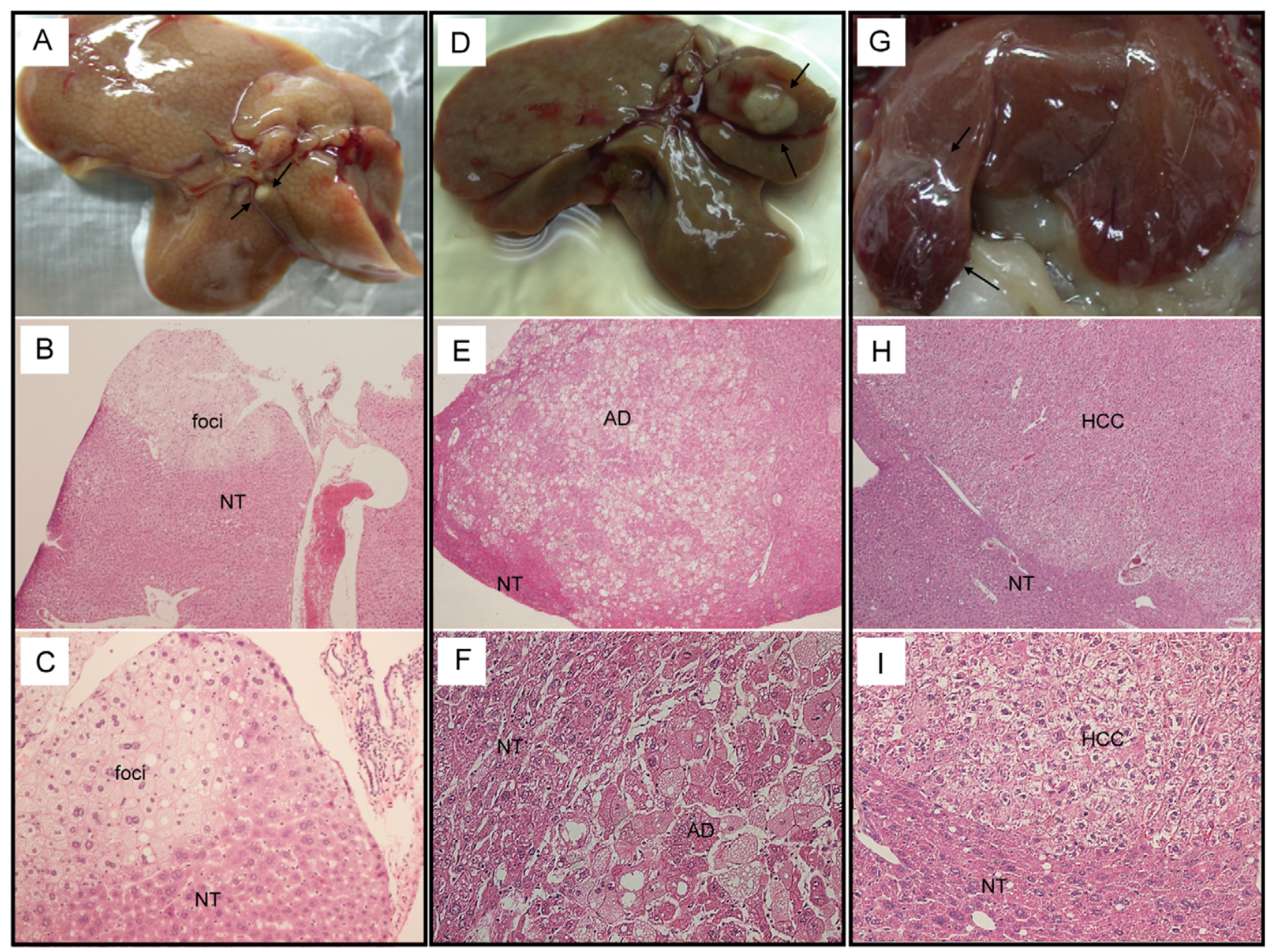

Figure 4. RBMY induced hepatic neoplastic changes in transgenic mice. (A-C) Pre-neoplastic lesion in a 15-month-old male mouse RF1-278. (D-F) Adenoma (AD) in a 24-month-old female mouse RF4-21. (G-I) HCC in a 21-month-old male mouse RF2-390. Magnification: B, E, H, $\times 50 ;$ C, F, I, $\times 200$.

doi:10.1371/journal.pone.0026948.g004 
Table 2. Incidence of hepatocarcinogenesis induced by DEN in RBMY transgenic and control mice.

\begin{tabular}{|c|c|c|c|c|c|}
\hline \multirow[t]{4}{*}{ Gender } & \multirow[t]{4}{*}{ RBMY } & \multirow{4}{*}{$\begin{array}{l}14 \text { wks } \\
\text { Incidence of } \\
\text { cancerous } \\
\text { lesions (No. mice) }\end{array}$} & \multirow{4}{*}{$\begin{array}{l}26 \text { wks } \\
\text { Incidence of } \\
\text { cancerous lesions } \\
\text { (No. mice) }\end{array}$} & \multicolumn{2}{|l|}{34 wks } \\
\hline & & & & \multirow{2}{*}{$\begin{array}{l}\text { Incidence of } \\
\text { cancerous lesions }\end{array}$} & \multirow{2}{*}{$\frac{\text { No. tumor }}{\geq 3 \mathrm{~mm}}$} \\
\hline & & & & & \\
\hline & & & & (No. Mice) & (No. mice) \\
\hline $\mathrm{F}$ & + & ND & $78 \%(7 / 9)^{* *}$ & $90 \%(9 / 10)^{* *}$ & $0(10)$ \\
\hline $\mathrm{F}$ & - & ND & $10 \%(1 / 10)$ & $23 \%(3 / 13)$ & $0(13)$ \\
\hline M & + & $86 \%(12 / 14)^{*}$ & $100 \%(5 / 5)$ & $100 \%(6 / 6)$ & $52(6)^{*}$ \\
\hline M & - & $42 \%(5 / 12)$ & $90 \%(9 / 10)$ & $100 \%(7 / 7)$ & 19 (7) \\
\hline
\end{tabular}

RBMY transgenic or control mice (14-day old) received a single intra-peritoneal injection of diethyl nitrosamine (DEN) $10 \mathrm{mg} / \mathrm{kg}$ of body weight. They were euthanized by cervical dislocation at 26 and 34 weeks for females, and at 14, 26, and 34 weeks for males after injection.

ND, not determined.

${ }^{*} p<0.05$;

${ }^{* *} p<0.01$, RBMY-positive vs. RBMY-negative mice; by Student's $t$ test.

doi:10.1371/journal.pone.0026948.t002

predominance of $\mathrm{HBV}$-related human HCG [16]. However, it cannot explain the gender disparity of non-HBV-related HCC. Similar ratio of RBMY expression in HBV-related, HCV-related, and non-viral-related male HCGs suggests that RBMY may be a common risk factor increasing male susceptibility for liver cancer. Our findings provide a novel mechanism interpreting the male predominance in all types of liver cancers through the $\mathrm{Y}$ chromosome-specific RBMY gene.

The AR45-specific exon 1B lies between the first and the second exons of the AR gene. Two hypothetical regulatory mechanisms for AR45 synthesis have been proposed, including a transcriptional control by a novel promoter upstream of exon $1 \mathrm{~B}$ or an alternative splicing event [13]. RBMY acts as a testis-specific splicing regulator and reportedly interacts with RNA-binding protein Sam68 in the testis [26]. Sam68 is a ubiquitous splicing regulator and also a downstream target of the Src signaling pathway $[28,29]$. It is thus far unclear whether or not RBMY either directly regulates AR45 transcription/splicing or via interacting with Sam68. In addition, Sam68 is considered an $\mathrm{AR}$ co-activator as it can modulate $\mathrm{AR}$ transcriptional activity in prostate cancers [25]. The Src signaling pathway also has been shown to be critical for the HBV X protein-mediated enhancement of AR function [30]. Whether RBMY is involved in the Sam68-mediated Src signaling pathway is an interesting issue to be studied in the future.

The oncogenicity of RBMY has been shown by the transformation of mouse fibroblast cell line NIH3T3 [24]. This study further demonstrates that RBMY enhances liver carcinogenesis in vivo in a transgenic mouse model. The reported spontaneous liver tumor growth rates are $3 \%$ and $0 \%$ in 24 month-old male and female wild type $\mathrm{FVB} / \mathrm{N}$ mice, respectively [31]. RBMY transgenic mice developed liver tumors in $8.7 \%(2 / 23)$ of male and $4.5 \%(1 / 22)$ of female mice older than 15 months, which are more than 2-fold higher than the spontaneous liver tumor incidences.

In the model of DEN-induced hepatocarcinogenesis, the significance of RBMY enhancing effect on cancerous lesion formation is observed as early as 14 weeks post-treatment in male transgenic mice. In addition, larger tumors (diameter $\geq 3 \mathrm{~mm}$ ) developed in transgenic male mice at 34 weeks, indicating RBMY enhancement on DEN-induced hepatocarcinogenesis. Even female RBMY transgenic mice also have significantly increased incidence of cancerous lesions at both 26 and 34 weeks posttreatment. Although the wild type female mice are resistant to
DEN carcinogenesis due to the protective effect of estrogenmediated inhibition of IL-6 production by Kupffer cells [32,33], the results here demonstrate the delivery and activation of a malespecific RBMY gene accelerated liver cancer development even in female transgenic mice.

In conclusion, RBMY knockdown elicits inhibitory effects on the transformation and anti-apoptotic abilities of the human hepatoma cell line HepG2. The inhibitory effect of RBMY on AR45 levels is demonstrated in RBMY knockdown HepG2 cells and RBMY over-expressing Hep3B and Huh7 cells. Therefore, the oncogenic mechanism of RBMY may be linked to its regulation of $\mathrm{AR}$ trans-activation activity by the increase of $\mathrm{AR} 45$ variant, the inhibitor of AR. AR45 expression detected in RBMY-expressing human male HCG is also significantly lower compared to non-expressing HCG tissues. Furthermore, RBMY exhibits tumor-promoting activity in vivo in a transgenic mice model and accelerates the development of neoplastic lesions in a diethylnitrosamine-induced hepatocarcinogenesis animal model. The carcinogenic effect in hepatoma cell line and human HCC tissues, along with in vivo tumor promotion in the transgenic mice, provides a novel role of RBMY as a male-specific oncogene to explain the male predominance of liver cancer.

\section{Supporting Information}

Table S1 Primer sequences in shRNA plasmid construction, semi-quantitative and real-time RT-PGR. (DOC)

\section{Acknowledgments}

The authors express their gratitude to Dr. Carol H. Miao of the Department of Biochemistry, University of Washington for the plasmid pBS-HCR-HPI-A, to Dr. Hui-Lin Wu of the Hepatitis Research Center, the National Taiwan University Hospital for the hepatoma cell line Huh7, and to Dr. Lih-Hwa Hwang of the Institute of Microbiology and Immunology, National Yang-Ming University for the helpful discussions in preparing this manuscript. The authors are also grateful for the support of the National Laboratory Animal Center in generating the transgenic mice used in this study.

\section{Author Contributions}

Conceived and designed the experiments: DJT MHC. Performed the experiments: DJT HYP SLL DSS SHH. Analyzed the data: YMJ HCH JFW YHN. Contributed reagents/materials/analysis tools: PHL HCH. Wrote the paper: DJT MHC. 


\section{References}

1. Bosch FX, Ribes J, Diaz M, Cleries R (2004) Primary liver cancer: worldwide incidence and trends. Gastroenterology 127: S5-S16.

2. El-Serag HB, Rudolph KL (2007) Hepatocellular carcinoma: epidemiology and molecular carcinogenesis. Gastroenterology 132: 2557-2576.

3. Yang JD, Roberts LR (2010) Hepatocellular carcinoma: A global view. Nat Rev Gastroenterol Hepatol 7: 448-458.

4. Yu MW, Cheng SW, Lin MW, Yang SY, Liaw YF, et al. (2000) Androgenreceptor gene CAG repeats, plasma testosterone levels, and risk of hepatitis Brelated hepatocellular carcinoma. J Natl Cancer Inst 92: 2023-2028.

5. Chang MH, Chen CJ, Lai MS, Hsu HM, Wu TC, et al. (1997) Universal hepatitis B vaccination in Taiwan and the incidence of hepatocellular carcinoma in children. Taiwan Childhood Hepatoma Study Group. N Engl J Med 336: 1855-1859.

6. Chang MH, Shau WY, Chen CJ, Wu TC, Kong MS, et al. (2000) Hepatitis B vaccination and hepatocellular carcinoma rates in boys and girls. JAMA 284: 3040-3042.

7. Kanda T, Steele R, Ray R, Ray RB (2008) Hepatitis G virus core protein augments androgen receptor-mediated signalling. J Virol 82: 11066-11072.

8. Ruggieri A, Barbati C, Malorni W (2010) Cellular and molecular mechanisms involved in hepatocellular carcinoma gender disparity. Int J Cancer 127: 499-504.

9. Kemp CJ, Leary CN, Drinkwater NR (1989) Promotion of murine hepatocarcinogenesis by testosterone is androgen receptor-dependent but not cell autonomous. Proc Natl Acad Sci U S A 86: 7505-7509.

10. Peng L, Malloy PJ, Wang J, Feldman D (2006) Growth inhibitory concentrations of androgens up-regulate insulin-like growth factor binding protein-3 expression via an androgen response element in LNCaP human prostate cancer cells. Endocrinology 147: 4599-4607.

11. Pandini G, Mineo R, Frasca F, Roberts CT, Jr., Marcelli M, et al. (2005) Androgens up-regulate the insulin-like growth factor-I receptor in prostate cancer cells. Cancer Res 65: 1849-1857.

12. Lee HJ, Chang G (2003) Recent advances in androgen receptor action. Cell Mol Life Sci 60: 1613-1622.

13. Ahrens-Fath I, Politz O, Geserick C, Haendler B (2005) Androgen receptor function is modulated by the tissue-specific AR45 variant. FEBS J 272: $74-84$.

14. Chamberlain NL, Driver ED, Miesfeld RL (1994) The length and location of CAG trinucleotide repeats in the androgen receptor $\mathrm{N}$-terminal domain affect transactivation function. Nucleic Acids Res 22: 3181-3186.

15. Wang SH, Yeh SH, Lin WH, Wang HY, Chen DS, et al. (2009) Identification of androgen response elements in the enhancer I of hepatitis $\mathrm{B}$ virus: a mechanism for sex disparity in chronic hepatitis B. Hepatology 50: 1392-1402.

16. Yang WJ, Chang CJ, Yeh SH, Lin WH, Wang SH, et al. (2009) Hepatitis B virus $\mathrm{X}$ protein enhances the transcriptional activity of the androgen receptor through c-Src and glycogen synthase kinase-3beta kinase pathways. Hepatology 49: 1515-1524.

17. Wu MH, Ma WL, Hsu CL, Chen YL, Ou JH, et al. (2010) Androgen receptor promotes hepatitis $\mathrm{B}$ virus-induced hepatocarcinogenesis through modulation of hepatitis B virus RNA transcription. Sci Transl Med 2: 32ra35.
18. Tsuei DJ, Chang MH, Chen PJ, Hsu TY, Ni YH (2002) Characterization of integration patterns and flanking cellular sequences of hepatitis $\mathrm{B}$ virus in childhood hepatocellular carcinomas. J Med Virol 68: 513-521.

19. Yin YH, Li YY, Qiao H, Wang HC, Yang XA, et al. (2005) TSPY is a cancer testis antigen expressed in human hepatocellular carcinoma. Br J Cancer 93: 458-463.

20. Elliott DJ, Millar MR, Oghene K, Ross A, Kiesewetter F, et al. (1997) Expression of RBM in the nuclei of human germ cells is dependent on a critical region of the $\mathrm{Y}$ chromosome long arm. Proc Natl Acad Sci U S A 94: 3848-3853.

21. Mahadevaiah SK, Odorisio T, Elliott DJ, Rattigan A, Szot M, et al. (1998) Mouse homologues of the human AZF candidate gene RBM are expressed in spermatogonia and spermatids, and map to a Y chromosome deletion interval associated with a high incidence of sperm abnormalities. Hum Mol Genet 7: 715-727.

22. Dreumont N, Bourgeois GF, Lejeune F, Liu Y, Ehrmann IE, et al. (2010) Human RBMY regulates germline-specific splicing events by modulating the function of the serine/arginine-rich proteins 9 G8 and Tra2-\{beta $\}$. J Cell Sci 123: $40-50$.

23. Liu Y, Bourgeois CF, Pang S, Kudla M, Dreumont N, et al. (2009) The germ cell nuclear proteins hnRNP G-T and RBMY activate a testis-specific exon. PLoS Genet 5: e1000707.

24. Tsuei DJ, Hsu HC, Lee PH, Jeng YM, Pu YS, et al. (2004) RBMY, a male germ cell-specific RNA-binding protein, activated in human liver cancers and transforms rodent fibroblasts. Oncogene 23: 5815-5822.

25. Rajan P, Gaughan L, Dalgliesh C, El-Sherif A, Robson CN, et al. (2008) The RNA-binding and adaptor protein Sam68 modulates signal-dependent splicing and transcriptional activity of the androgen receptor. J Pathol 215: 67-77.

26. Venables JP, Vernet C, Chew SL, Elliott DJ, Cowmeadow RB, et al. (1999) TSTAR/ETOILE: a novel relative of SAM68 that interacts with an RNA-binding protein implicated in spermatogenesis. Hum Mol Genet 8: 959-969.

27. Angulo P, Lindor KD (2002) Non-alcoholic fatty liver disease. J Gastroenterol Hepatol 17 Suppl: S186-190.

28. Fumagalli S, Totty NF, Hsuan JJ, Courtneidge SA (1994) A target for Src in mitosis. Nature 368: 871-874.

29. Matter N, Herrlich P, Konig H (2002) Signal-dependent regulation of splicing via phosphorylation of Sam68. Nature 420: 691-695.

30. Chiu CM, Yeh SH, Chen PJ, Kuo TJ, Chang CJ, et al. (2007) Hepatitis B virus $\mathrm{X}$ protein enhances androgen receptor-responsive gene expression depending on androgen level. Proc Natl Acad Sci U S A 104: 2571-2578.

31. Mahler JF, Stokes W, Mann PC, Takaoka M, Maronpot RR (1996) Spontaneous lesions in aging FVB/N mice. Toxicol Pathol 24: 710-716.

32. Naugler WE, Sakurai T, Kim S, Maeda S, Kim K, et al. (2007) Gender disparity in liver cancer due to sex differences in MyD88-dependent IL-6 production. Science 317: 121-124.

33. Prieto J (2008) Inflammation, HCC and sex: IL-6 in the centre of the triangle. J Hepatol 48: 380-381. 